\title{
Table de concordance
}

\section{Q OpenEdition}

1 Journals

Édition électronique

URL : http://journals.openedition.org/ccs/1817

DOI : $10.4000 /$ ccs. 1817

ISSN : 2558-782X

Éditeur :

Presses universitaires de Rennes, Association des lecteurs de Claude Simon

\section{Édition imprimée}

Date de publication : 30 août 2018

Pagination : 195-198

ISBN : 978-2-7535-7489-2

ISSN : $1774-9425$

Référence électronique

"Table de concordance », Cahiers Claude Simon [En ligne], 13 | 2018, mis en ligne le 30 août 2019, consulté le 20 février 2020. URL : http://journals.openedition.org/ccs/1817; DOI : 10.4000/ccs.1817

Cahiers Claude Simon 


\section{TABLE DE CONCORDANCE}

Le Jardin des Plantes compte à ce jour deux éditions : la première de 1997 aux Editions de Minuit, la seconde de 2006 dans le premier volume des cuvres de Claude Simon publiées dans la "Bibliothèque de la Pléiade " (Gallimard, 2006) sous la direction d'Alastair B. Duncan (notice et notes de Jean H. Duffy pour ce roman).

Les références au Jardin des Plantes données dans ce numéro des Cahiers renvoient toutes à la pagination du roman dans la Pléiade. Pour faciliter les reports d'une édition à l'autre, nous proposons cette table de concordance qui met en regard chaque début de page de l'édition Minuit avec la localisation du même passage dans la Pléiade. 


\begin{tabular}{|c|c|}
\hline Minuit & Pléiade \\
\hline 7 & 903 \\
\hline 8 & 904 \\
\hline 9 & 905 \\
\hline 10 & 906 \\
\hline 11 & 907 \\
\hline 12 & 907 \\
\hline 13 & 908 \\
\hline 14 & 909 \\
\hline 15 & 910 \\
\hline 16 & 910 \\
\hline 17 & 911 \\
\hline 18 & 912 \\
\hline 19 & 913 \\
\hline 20 & 913 \\
\hline 21 & 914 \\
\hline 22 & 915 \\
\hline 23 & 916 \\
\hline 24 & 916 \\
\hline 25 & 917 \\
\hline 26 & 918 \\
\hline 27 & 919 \\
\hline 28 & 919 \\
\hline 29 & 920 \\
\hline 30 & 921 \\
\hline 31 & 922 \\
\hline 32 & 922 \\
\hline 33 & 923 \\
\hline 34 & 924 \\
\hline 35 & 924 \\
\hline 36 & 925 \\
\hline 37 & 926 \\
\hline 38 & 927 \\
\hline 39 & 927 \\
\hline 40 & 928 \\
\hline 41 & 929 \\
\hline
\end{tabular}

\begin{tabular}{|c|c|}
\hline 42 & 930 \\
\hline 43 & 930 \\
\hline 44 & 931 \\
\hline 45 & 932 \\
\hline 46 & 932 \\
\hline 47 & 933 \\
\hline 48 & 934 \\
\hline 49 & 935 \\
\hline 50 & 935 \\
\hline 51 & 936 \\
\hline 52 & 937 \\
\hline 53 & 938 \\
\hline 54 & 938 \\
\hline 55 & 939 \\
\hline 56 & 940 \\
\hline 57 & 941 \\
\hline 58 & 941 \\
\hline 59 & 942 \\
\hline 60 & 943 \\
\hline 61 & 943 \\
\hline 62 & 944 \\
\hline 63 & 945 \\
\hline 64 & 946 \\
\hline 65 & 946 \\
\hline 66 & 947 \\
\hline 67 & 948 \\
\hline 68 & 949 \\
\hline 69 & 949 \\
\hline 70 & 950 \\
\hline 71 & 951 \\
\hline 72 & 951 \\
\hline 73 & 952 \\
\hline 74 & 953 \\
\hline 75 & 954 \\
\hline 76 & 954 \\
\hline 77 & 955 \\
\hline
\end{tabular}

\begin{tabular}{|c|c|}
\hline 78 & 956 \\
\hline 79 & 956 \\
\hline 80 & 957 \\
\hline 81 & 958 \\
\hline 82 & 959 \\
\hline 83 & 959 \\
\hline 84 & 960 \\
\hline 85 & 961 \\
\hline 86 & 962 \\
\hline 87 & 962 \\
\hline 88 & 963 \\
\hline 89 & 964 \\
\hline 90 & 965 \\
\hline 91 & 965 \\
\hline 92 & 966 \\
\hline 93 & 967 \\
\hline 94 & 967 \\
\hline 95 & 968 \\
\hline 96 & 969 \\
\hline 97 & 970 \\
\hline 98 & 970 \\
\hline 99 & 971 \\
\hline 100 & 972 \\
\hline 101 & 972 \\
\hline 102 & 973 \\
\hline 103 & 974 \\
\hline 104 & 975 \\
\hline 105 & 975 \\
\hline 106 & 976 \\
\hline 107 & 977 \\
\hline 108 & 978 \\
\hline 109 & 978 \\
\hline 110 & 979 \\
\hline 111 & 980 \\
\hline 112 & 980 \\
\hline 113 & 981 \\
\hline
\end{tabular}

\begin{tabular}{|c|c|}
\hline 114 & 982 \\
\hline 115 & 983 \\
\hline 116 & 983 \\
\hline 117 & 984 \\
\hline 118 & 985 \\
\hline 119 & 986 \\
\hline 120 & 986 \\
\hline 121 & 987 \\
\hline 122 & 988 \\
\hline 123 & 988 \\
\hline 124 & 989 \\
\hline 125 & 990 \\
\hline 126 & 991 \\
\hline 127 & 991 \\
\hline 128 & 992 \\
\hline 129 & 993 \\
\hline 130 & 994 \\
\hline 131 & 994 \\
\hline 132 & 995 \\
\hline 133 & 996 \\
\hline 134 & 996 \\
\hline 135 & 997 \\
\hline 136 & 998 \\
\hline 137 & 999 \\
\hline 138 & 999 \\
\hline 139 & 1000 \\
\hline 140 & 1001 \\
\hline 141 & 1002 \\
\hline 142 & 1002 \\
\hline 143 & 1003 \\
\hline 144 & 1004 \\
\hline 145 & 1004 \\
\hline 146 & 1005 \\
\hline 147 & 1006 \\
\hline 148 & 1007 \\
\hline 149 & 1007 \\
\hline
\end{tabular}


TABLE DE CONCORDANCE

\begin{tabular}{|c|c|}
\hline Minuit & Pléiade \\
\hline 150 & 1008 \\
\hline 151 & 1009 \\
\hline 152 & 1010 \\
\hline 153 & 1010 \\
\hline 154 & 1011 \\
\hline 155 & 1013 \\
\hline 156 & 1014 \\
\hline 157 & 1015 \\
\hline 158 & 1015 \\
\hline 159 & 1016 \\
\hline 160 & 1017 \\
\hline 161 & 1017 \\
\hline 162 & 1018 \\
\hline 163 & 1019 \\
\hline 164 & 1020 \\
\hline 165 & 1020 \\
\hline 166 & 1021 \\
\hline 167 & 1022 \\
\hline 168 & 1023 \\
\hline 169 & 1023 \\
\hline 170 & 1024 \\
\hline 171 & 1025 \\
\hline 172 & 1025 \\
\hline 173 & 1026 \\
\hline 174 & 1027 \\
\hline 175 & 1028 \\
\hline 176 & 1028 \\
\hline 177 & 1029 \\
\hline 178 & 1030 \\
\hline 179 & 1031 \\
\hline 180 & 1031 \\
\hline 181 & 1032 \\
\hline 182 & 1033 \\
\hline 183 & 1034 \\
\hline 184 & 1034 \\
\hline
\end{tabular}

\begin{tabular}{|c|c|}
\hline 185 & 1035 \\
\hline 186 & 1036 \\
\hline 187 & 1036 \\
\hline 188 & 1037 \\
\hline 189 & 1038 \\
\hline 190 & 1039 \\
\hline 191 & 1039 \\
\hline 192 & 1040 \\
\hline 193 & 1041 \\
\hline 194 & 1042 \\
\hline 195 & 1042 \\
\hline 196 & 1043 \\
\hline 197 & 1044 \\
\hline 198 & 1045 \\
\hline 199 & 1045 \\
\hline 200 & 1046 \\
\hline 201 & 1047 \\
\hline 202 & 1047 \\
\hline 203 & 1048 \\
\hline 204 & 1049 \\
\hline 205 & 1050 \\
\hline 206 & 1050 \\
\hline 207 & 1051 \\
\hline 208 & 1052 \\
\hline 209 & 1052 \\
\hline 210 & 1053 \\
\hline 211 & 1054 \\
\hline 212 & 1055 \\
\hline 213 & 1055 \\
\hline 214 & 1056 \\
\hline 215 & 1057 \\
\hline 216 & 1057 \\
\hline 217 & 1058 \\
\hline 218 & 1059 \\
\hline 219 & 1061 \\
\hline 220 & 1062 \\
\hline
\end{tabular}

\begin{tabular}{|c|c|}
\hline 221 & 1063 \\
\hline 222 & 1063 \\
\hline 223 & 1064 \\
\hline 224 & 1065 \\
\hline 225 & 1066 \\
\hline 226 & 1066 \\
\hline 227 & 1067 \\
\hline 228 & 1068 \\
\hline 229 & 1068 \\
\hline 230 & 1069 \\
\hline 231 & 1070 \\
\hline 232 & 1071 \\
\hline 233 & 1071 \\
\hline 234 & 1072 \\
\hline 235 & 1073 \\
\hline 236 & 1073 \\
\hline 237 & 1074 \\
\hline 238 & 1075 \\
\hline 239 & 1076 \\
\hline 240 & 1076 \\
\hline 241 & 1077 \\
\hline 242 & 1078 \\
\hline 243 & 1078 \\
\hline 244 & 1079 \\
\hline 245 & 1080 \\
\hline 246 & 1081 \\
\hline 247 & 1081 \\
\hline 248 & 1082 \\
\hline 249 & 1083 \\
\hline 250 & 1084 \\
\hline 251 & 1084 \\
\hline 252 & 1085 \\
\hline 253 & 1086 \\
\hline 254 & 1086 \\
\hline 255 & 1087 \\
\hline 256 & 1088 \\
\hline
\end{tabular}

\begin{tabular}{|c|c|}
\hline 257 & 1089 \\
\hline 258 & 1089 \\
\hline 259 & 1090 \\
\hline 260 & 1091 \\
\hline 261 & 1091 \\
\hline 262 & 1092 \\
\hline 263 & 1093 \\
\hline 264 & 1094 \\
\hline 265 & 1094 \\
\hline 266 & 1095 \\
\hline 267 & 1096 \\
\hline 268 & 1097 \\
\hline 269 & 1097 \\
\hline 270 & 1098 \\
\hline 271 & 1099 \\
\hline 272 & 1100 \\
\hline 273 & 1100 \\
\hline 274 & 1101 \\
\hline 275 & 1102 \\
\hline 276 & 1103 \\
\hline 277 & 1103 \\
\hline 278 & 1104 \\
\hline 279 & 1105 \\
\hline 280 & 1106 \\
\hline 281 & 1106 \\
\hline 282 & 1107 \\
\hline 283 & 1108 \\
\hline 284 & 1108 \\
\hline 285 & 1109 \\
\hline 286 & 1110 \\
\hline 287 & 1111 \\
\hline 288 & 1111 \\
\hline 289 & 1112 \\
\hline 290 & 1113 \\
\hline 291 & 1114 \\
\hline 292 & 1114 \\
\hline
\end{tabular}




\begin{tabular}{|c|c|}
\hline Minuit & Pléiade \\
\hline 293 & 1115 \\
\hline 294 & 1116 \\
\hline 295 & 1116 \\
\hline 296 & 1117 \\
\hline 297 & 1118 \\
\hline 298 & 1119 \\
\hline 299 & 1119 \\
\hline 300 & 1120 \\
\hline 301 & 1121 \\
\hline 302 & 1122 \\
\hline 303 & 1122 \\
\hline 304 & 1123 \\
\hline 305 & 1124 \\
\hline 306 & 1124 \\
\hline 307 & 1125 \\
\hline 308 & 1126 \\
\hline 309 & 1127 \\
\hline 310 & 1127 \\
\hline 311 & 1128 \\
\hline 312 & 1129 \\
\hline 313 & 1130 \\
\hline 314 & 1130 \\
\hline 315 & 1131 \\
\hline 316 & 1132 \\
\hline 317 & 1133 \\
\hline 318 & 1134 \\
\hline 319 & 1135 \\
\hline 320 & 1135 \\
\hline 321 & 1136 \\
\hline 322 & 1137 \\
\hline 323 & 1138 \\
\hline 324 & 1138 \\
\hline 325 & 1139 \\
\hline 326 & 1140 \\
\hline 327 & 1140 \\
\hline
\end{tabular}

\begin{tabular}{|c|c|}
\hline 328 & 1141 \\
\hline 329 & 1142 \\
\hline 330 & 1143 \\
\hline 331 & 1143 \\
\hline 332 & 1144 \\
\hline 333 & 1145 \\
\hline 334 & 1146 \\
\hline 335 & 1146 \\
\hline 336 & 1147 \\
\hline 337 & 1148 \\
\hline 338 & 1149 \\
\hline 339 & 1149 \\
\hline 340 & 1150 \\
\hline 341 & 1151 \\
\hline 342 & 1151 \\
\hline 343 & 1152 \\
\hline 344 & 1153 \\
\hline 345 & 1154 \\
\hline 346 & 1154 \\
\hline 347 & 1155 \\
\hline 348 & 1156 \\
\hline 349 & 1157 \\
\hline 350 & 1157 \\
\hline 351 & 1158 \\
\hline 352 & 1159 \\
\hline 353 & 1160 \\
\hline 354 & 1160 \\
\hline 355 & 1161 \\
\hline 356 & 1162 \\
\hline 357 & 1162 \\
\hline 358 & 1163 \\
\hline 359 & 1164 \\
\hline 360 & 1165 \\
\hline 361 & 1165 \\
\hline 362 & 1166 \\
\hline 363 & 1167 \\
\hline
\end{tabular}

\begin{tabular}{|l|l|}
\hline 364 & 1167 \\
\hline 365 & 1168 \\
\hline 366 & 1169 \\
\hline 367 & 1170 \\
\hline 368 & 1170 \\
\hline 369 & 1171 \\
\hline 370 & 1172 \\
\hline 371 & 1173 \\
\hline 372 & 1173 \\
\hline 373 & 1174 \\
\hline 374 & 1175 \\
\hline 375 & 1176 \\
\hline 376 & 1176 \\
\hline 377 & 1177 \\
\hline 378 & 1178 \\
\hline
\end{tabular}

